\title{
vsFilt: A Tool to Improve Virtual Screening by Structural Filtration of Docking Poses
}

Irina V. Gushchina,,$^{\dagger}$ Aleksandra M. Polenova, ${ }^{\S}$ Dmitry A. Suplatov, ${ }^{\dagger}$ Vytas K. Švedas, ${ }^{\dagger}$ and Dmitry

$$
\text { K. Nilov }{ }^{*},+
$$

$\dagger$ Lomonosov Moscow State University, Belozersky Institute of Physicochemical Biology, Lenin hills 1, bldg. 40, 119991 Moscow, Russia

$\S$ Lomonosov Moscow State University, Faculty of Medicine, Lomonosov Ave. 27, bldg. 1, 119991 Moscow, Russia

*E-mail: nilov@belozersky.msu.ru 

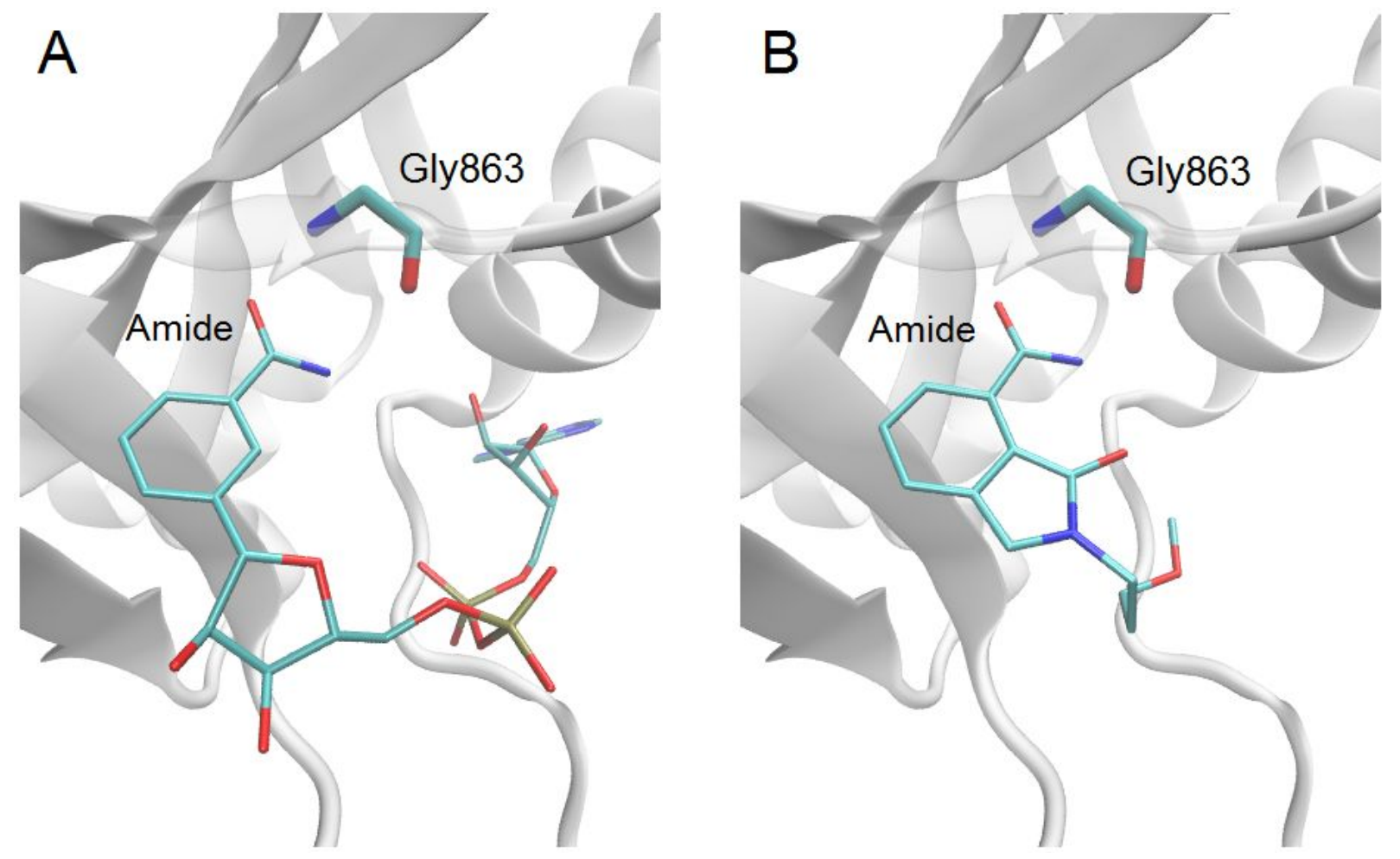

Figure S1. Binding of ligands in the PARP-1 active site. (A) Substrate (NAD ${ }^{+}$) analogue; PDB ID 6bhv. ${ }^{1}$ PARP-1 catalyzes the synthesis of a signal polymer, poly(ADP-ribose), from NAD ${ }^{+}$ molecules whose amide group specifically binds to Gly863 residue. (B) Inhibitor; 4zzz. ${ }^{2}$ PARP-1 inhibitors bind similarly to the $\mathrm{NAD}^{+}$nicotinamide moiety forming two hydrogen bonds with Gly863. This characteristic interaction could be taken as an effective criterion for the identification of new potent binders. 
Basic operations with the 3D-viewer: Left-click-and-hold and then move your mouse to rotate the structure, Shift + Left-click-and-hold + Mouse Up/Down to zoom in and out. Basic operations with the 3D-viewer: Left-click-and-hold and then move your mouse to rotate the structure, Shift + Left-click-and-hold + Mouse Up/Down to zoom in and out,
Ctrl + Right-click-and-hold + Mouse Up/Down/Left/Right to move the structure in the viewer, Right-click for more options. Hold mouse pointer over selected amino acid for one 作

Quick hints: Each ligand and its interactions with the protein residues that comply with the filtering rules can be visualized individually by clicking on the respective Quick hints: Each ligand and its interactions with the protein residues that comply with the filtering rules can be visualized individually by clicking on the respective checkbox, or loaded all at once by using the buttons Toggle all ligands (i.e., all ligands that passed the structural filtration will be shown in the 3D-viewer), Toggle residues restore all rows use the Restore all rows button. To highlight a ligand (row) in the table click on any cell (i.e., this feature can help to work with large tables).

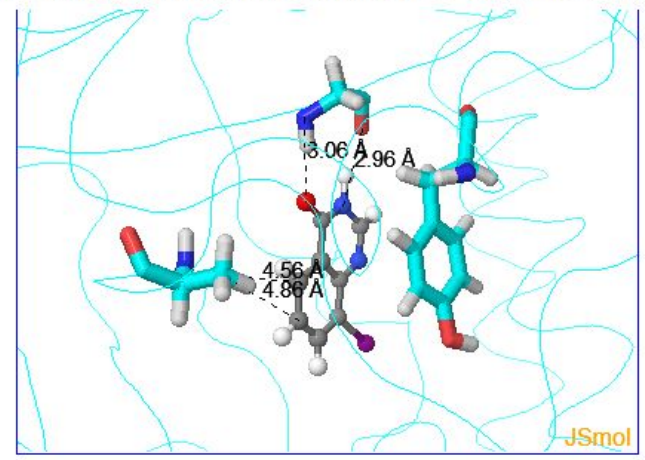

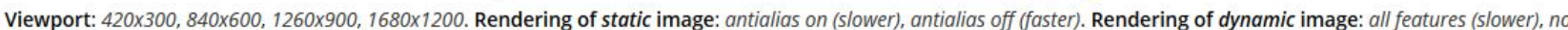
antialiasing, no translucency, surfaces dotted, cartoons as trace, geosurfaces as dots, ellipsoids as dots, wireframe only (faster).

Last action with the 3D-viewer: you have enabled ligand ZINC26894394 ranked \#1

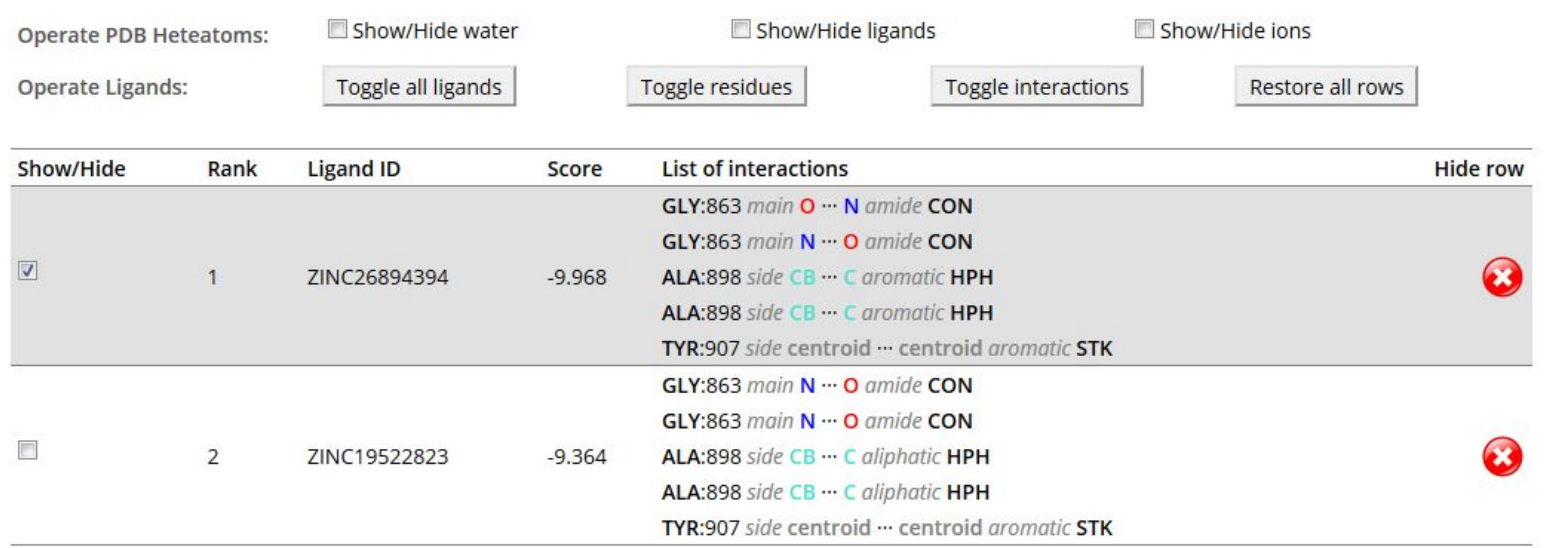

Figure S2. On-line analysis page of vsFilt. Each ligand and its interactions with the protein residues that meet the structural criteria can be visualized individually by clicking on the respective checkbox, or loaded all at once by using the buttons "Toggle all ligands", "Toggle residues", and "Toggle interactions". 
Table S1. Distance criteria for functional group interactions in vsFilt.

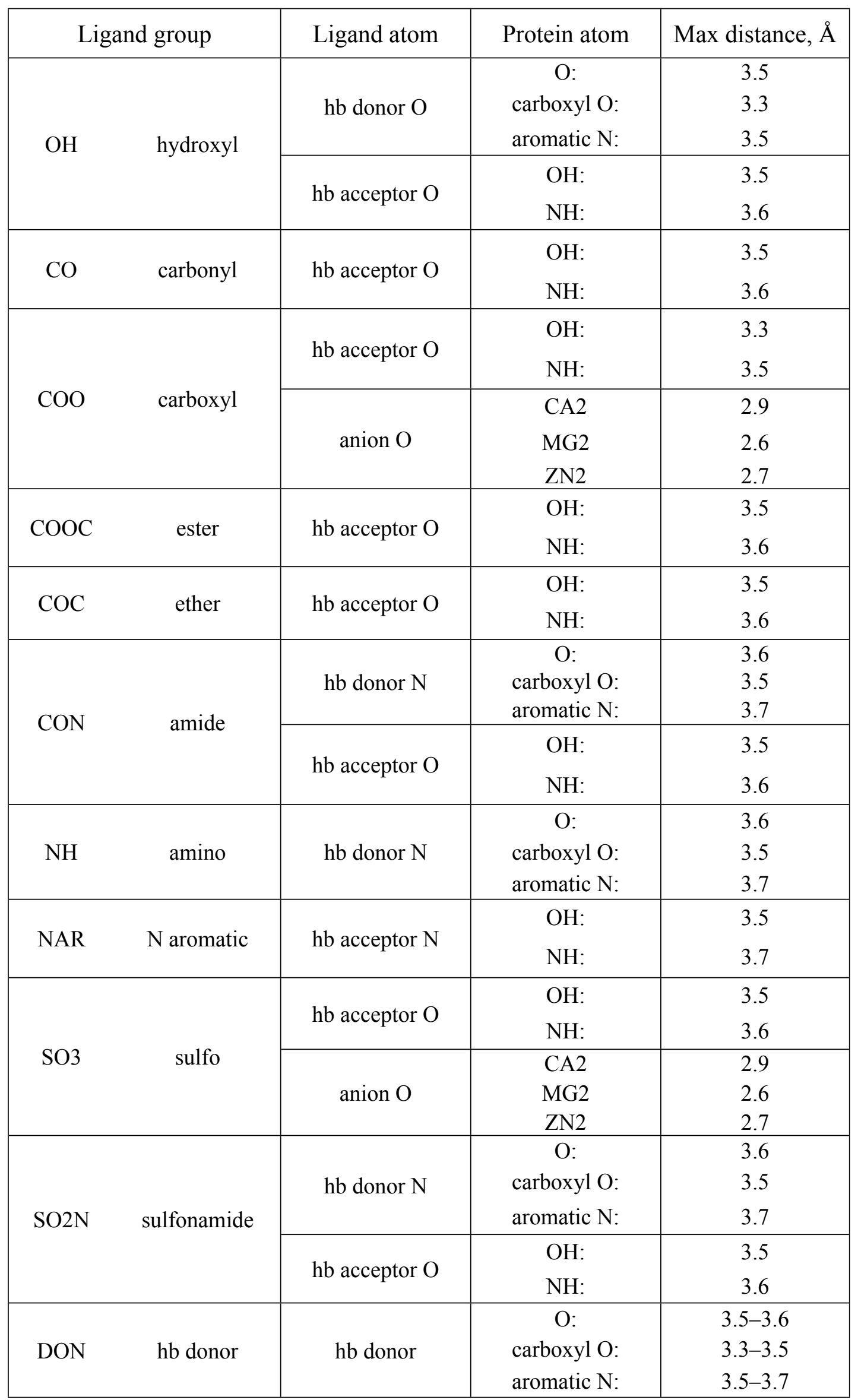




\begin{tabular}{|c|c|c|c|c|}
\hline $\mathrm{ACC}$ & hb acceptor & hb acceptor & $\begin{array}{l}\mathrm{OH}: \\
\mathrm{NH}:\end{array}$ & $\begin{array}{l}3.3-3.5 \\
3.5-3.7\end{array}$ \\
\hline \multirow{2}{*}{ HAL } & \multirow{2}{*}{ halo } & $\mathrm{Cl}$ & carbonyl O: & 3.9 \\
\hline & & $\mathrm{Br}, \mathrm{I}$ & carbonyl O: & 4.0 \\
\hline \multirow{4}{*}{$\mathrm{HPH}$} & \multirow{4}{*}{ hydrophobic } & aliphatic $\mathrm{C}$ & $\begin{array}{l}\text { aliphatic C } \\
\text { aromatic C }\end{array}$ & $\begin{array}{l}4.9 \\
4.9 \\
\end{array}$ \\
\hline & & aromatic $\mathrm{C}$ & $\begin{array}{l}\text { aliphatic C } \\
\text { aromatic C }\end{array}$ & $\begin{array}{l}4.9 \\
4.3 \\
\end{array}$ \\
\hline & & $\mathrm{F}$ & aliphatic C & 4.4 \\
\hline & & $\mathrm{Cl}$ & aliphatic C & 4.8 \\
\hline STK & aromatic & centroid $^{*}$ & centroid $^{* *}$ & 4.5 \\
\hline PIC & aromatic & aromatic $\mathrm{C}$ & guanidinium $\mathrm{C}$ & 4.5 \\
\hline
\end{tabular}

${ }^{*}$ Geometric center of ligand aromatic rings.

${ }^{* *}$ Geometric center of Phe/Tyr/His/Trp aromatic rings.

Table S2. PARP-1 inhibitor scaffolds selected by structural filtration of docked ZINC compounds with vsFilt. The number of selected ligands corresponding to each scaffold is indicated. The examples of selected compounds: ZINC26840105, ${ }^{3}$ ZINC36690025, ${ }^{4}$ ZINC72190317. ${ }^{5}$

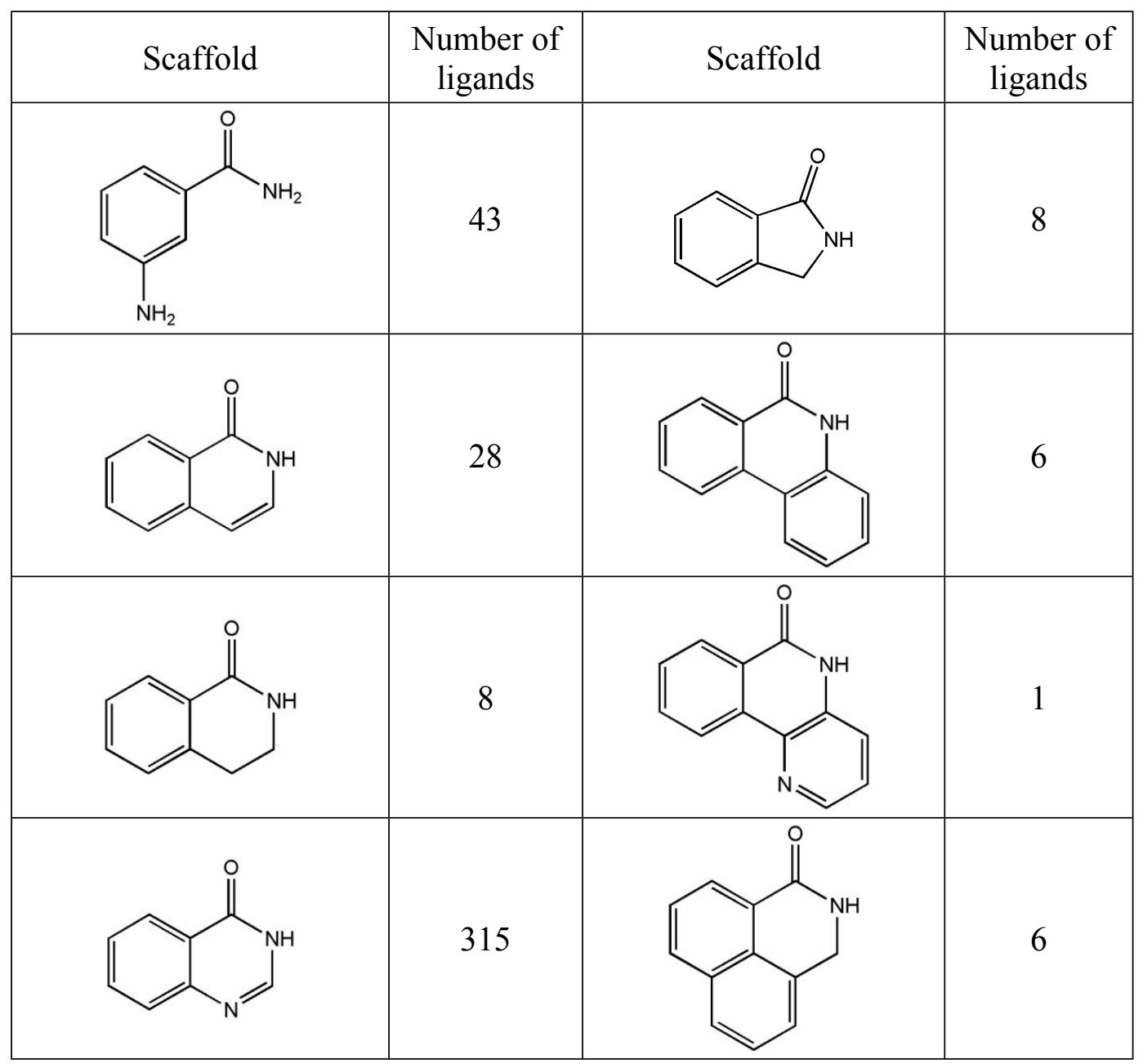




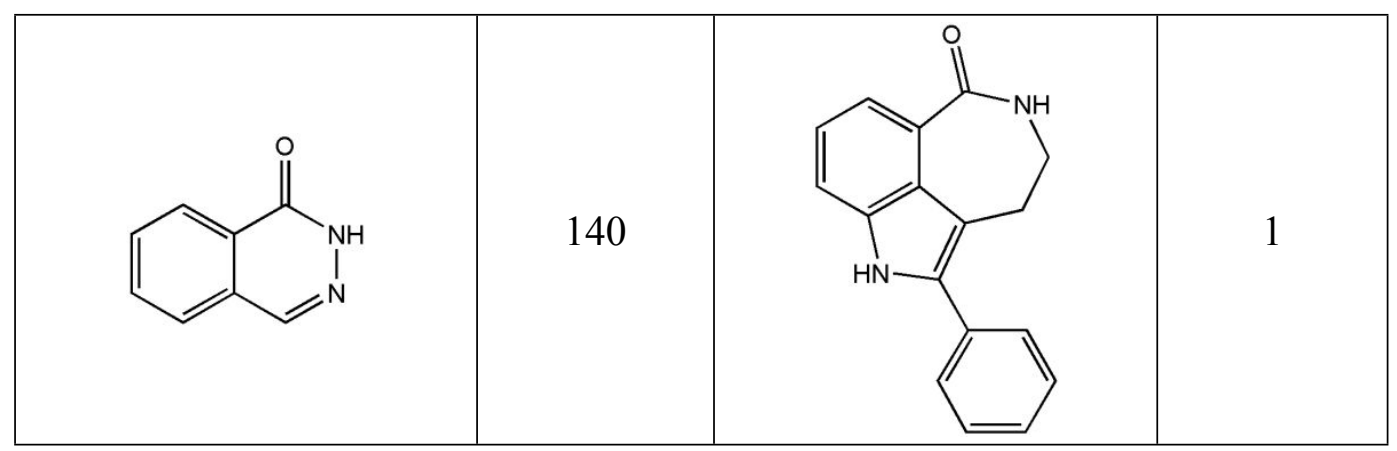

Table S3. Distance and angle characteristics of ZINC26894394 ligand in the active site of PARP-1 model meet the structural criteria applied by vsFilt.

\begin{tabular}{|c|c|c|}
\hline Interaction & Distance, $\AA$ & Distance criteria, $\AA$ \\
\hline LIG:NH:H $\cdots$ Gly863:O & 2.96 & $\leq 3.1$ \\
\hline LIG:CO:O $\cdots$ Gly863:H & 3.06 & $\leq 3.1$ \\
\hline LIG:C arom $^{\cdots}$ Ala898:CB & 4.56 & $\leq 4.9$ \\
\hline $\begin{array}{c}\text { C(LIG aromatic ring) } \cdots \\
\text { C(Tyr907 benzene ring) }\end{array}$ & 4.49 & $\leq 4.5$ \\
\hline Interaction & Angle, deg & Angle criteria, deg \\
\hline LIG:NH:N $\cdots$ LIG:NH:H $\cdots$ Gly863:O & 159.6 & $\geq 150$ \\
\hline LIG:CO:O $\cdots$ Gly863:H $\cdots$ Gly863:N & 169.4 & $\geq 150$ \\
\hline
\end{tabular}

\section{Description of the $<$ ID $>$ and $<$ SCORE $>$ items in the SDF file}

SDF is a widely used structure-data file format, and its feature is the ability to introduce associated data items denoted as follows:

$>\quad<I D>$

STK381370

vsFilt is designed to read IDs of library ligands from the $<\mathrm{ID}>$ item introduced in compound libraries such as Vitas-M (https://vitasmlab.biz) or ChemBridge (https://www.chembridge.com). If the name of the item containing ligand ID is other than $<$ ID $>$, it should be renamed. If ligand ID is given on a separate line outside any associated data item, such as in ZINC (https://zinc.docking.org) ${ }^{6}$, it should be copied to $<\mathrm{ID}>$; a simple example script to perform this task is provided below. Otherwise, vsFilt automatically introduces $<$ ID $>$ item containing a serial number of a ligand in the input SDF file. Another SDF item that could be used by vsFilt is $<$ SCORE $>$ containing any information useful for ligand ranking (e.g., calculated $\Delta G$ ). If $<\operatorname{SCORE}>$ is introduced, vsFilt sorts ligands by the $<$ SCORE $>$ value and writes scores to the output TXT file. 
Example of a Perl script for introducing the $<$ ID $>$ field into SDF file of ZINC:

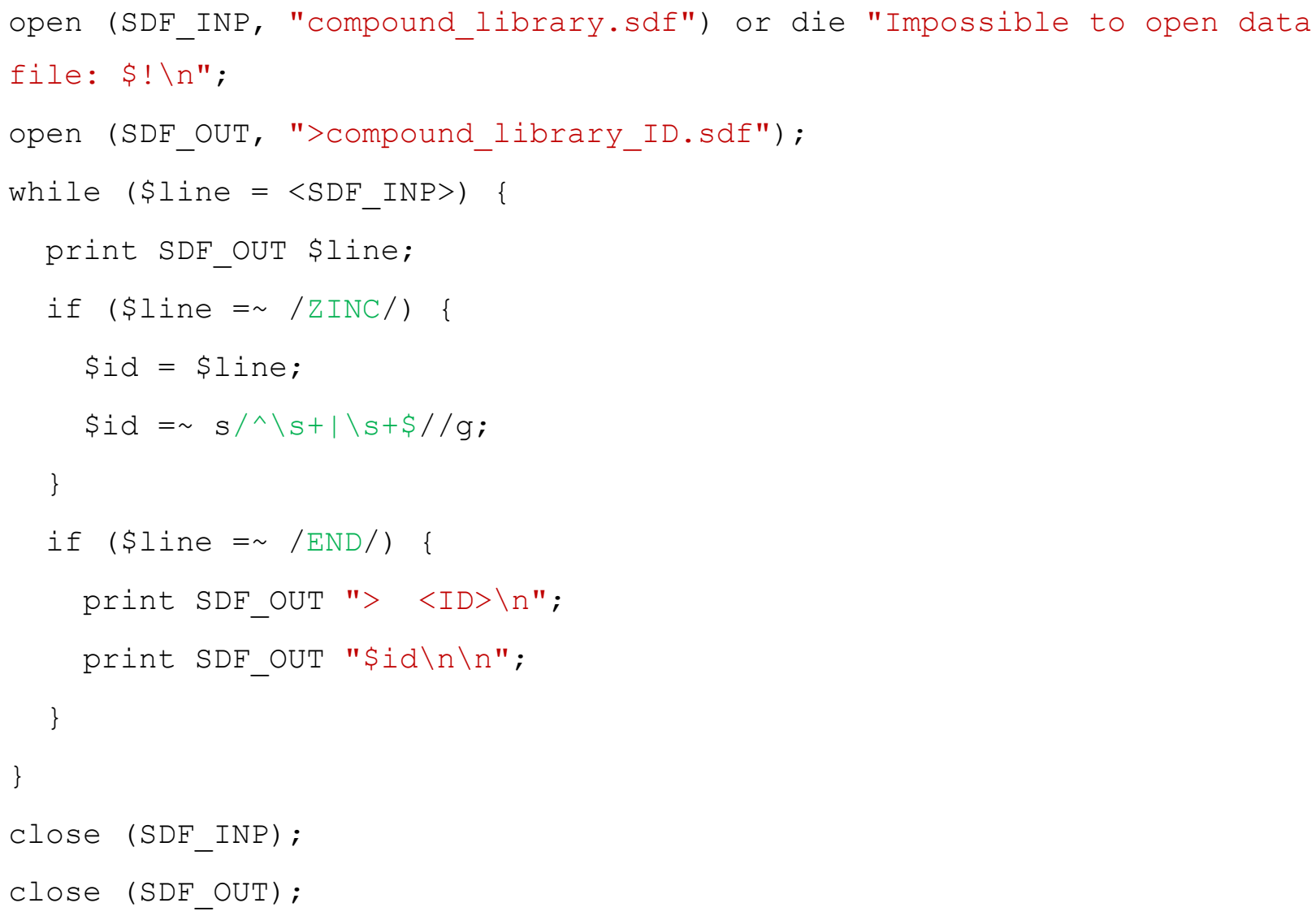

\section{Description of the PARP-1 model preparation}

The molecular model of catalytic domain of human PARP-1 was built on the basis of the 4zzz crystal structure ${ }^{2}$ using AmberTools15 and Amber $14 .{ }^{7}$ Hydrogen atoms were added to the protein structure, and then it was solvated by a $12 \AA$-thick layer of TIP3P water. The energy minimization (2500 steps of the steepest descent algorithm +2500 steps of the conjugate gradient algorithm) was performed to optimize the positions of hydrogen atoms. During the minimization, the protein molecule was described by the $f f 14 S B$ force field, ${ }^{8}$ and the heavy protein atoms were kept fixed by positional restraints of $2 \mathrm{kcal} /\left(\mathrm{mol} \cdot \AA^{2}\right)$. The non-bonded cut-off distance was $10 \AA$, and the particle mesh Ewald method was used to handle long-range electrostatic interactions. The water molecules were removed from the optimized system to obtain the protein model for molecular docking.

\section{References}

(1) Langelier, M. F.; Zandarashvili, L.; Aguiar, P. M.; Black, B. E.; Pascal, J. M. NAD ${ }^{+}$analog reveals PARP-1 substrate-blocking mechanism and allosteric communication from catalytic center to DNA-binding domains. Nat. Commun. 2018, 9, 844. 
(2) Papeo, G.; Posteri, H.; Borghi, D.; Busel, A. A.; Caprera, F.; Casale, E.; Ciomei, M.; Cirla, A.; Corti, E.; D'Anello, M.; Fasolini, M.; Forte, B.; Galvani, A.; Isacchi, A.; Khvat, A.; Krasavin, M. Y.; Lupi, R.; Orsini, P.; Perego, R.; Pesenti, E.; Pezzetta, D.; Rainoldi, S.; Riccardi-Sirtori, F.; Scolaro, A.; Sola, F.; Zuccotto, F.; Felder, E.R.; Donati, D.; Montagnoli, A. Discovery of 2-[1-(4,4Difluorocyclohexyl)piperidin-4-yl]-6-fluoro-3-oxo-2,3-dihydro-1H-isoindole-4-carboxamide (NMS-P118): A Potent, Orally Available, and Highly Selective PARP-1 Inhibitor for Cancer Therapy. J. Med. Chem. 2015, 58, 6875-6898.

(3) Ferraris, D.; Ko, Y. S.; Pahutski, T.; Ficco, R. P.; Serdyuk, L.; Alemu, C.; Bradford, C.; Chiou, T.; Hoover, R.; Huang, S.; Lautar, S.; Liang, S.; Lin, Q.; Lu, M. X.; Mooney, M.; Morgan, L.; Qian, Y.; Tran, S.; Williams, L. R.; Wu, Q. Y.; Zhang, J.; Zou, Y.; Kalish, V. Design and Synthesis of Poly ADP-ribose polymerase-1 Inhibitors. 2. Biological Evaluation of aza-5[H]-phenanthridin-6ones as Potent, Aqueous-Soluble Compounds for the Treatment of Ischemic Injuries. J. Med. Chem. 2003, 46, 3138-3151.

(4) Moroni, F.; Formentini, L.; Gerace, E.; Camaioni, E.; Pellegrini-Giampietro, D. E.; Chiarugi, A.; Pellicciari, R. Selective PARP-2 Inhibitors Increase Apoptosis in Hippocampal Slices but Protect Cortical Cells in Models of Post-Ischaemic Brain Damage. Br. J. Pharmacol. 2009, 157, 854-862.

(5) Thomas, H. D.; Calabrese, C. R.; Batey, M. A.; Canan, S.; Hostomsky, Z.; Kyle, S.; Maegley, K. A.; Newell, D. R.; Skalitzky, D.; Wang, L. Z.; Webber, S. E.; Curtin, N. J. Preclinical Selection of a Novel poly(ADP-ribose) Polymerase Inhibitor for Clinical Trial. Mol. Cancer Ther., 2007, 6, 945-956.

(6) Irwin, J. J.; Sterling, T.; Mysinger, M. M.; Bolstad, E. S.; Coleman, R. G. ZINC: a Free Tool to Discover Chemistry for Biology. J. Chem. Inf. Model. 2012, 52, 1757-1768.

(7) Case, D. A.; Berryman, J. T.; Betz, R. M.; Cerutti, D. S.; Cheatham, T. E. 3rd; Darden, T. A.; Duke, R. E.; Giese, T. J.; Gohlke, H.; Goetz, A. W.; Homeyer, N.; Izadi, S.; Janowski, P.; Kaus, J.; Kovalenko, A.; Lee, T. S.; LeGrand, S.; Li, P.; Luchko, T.; Luo, R.; Madej, B.; Merz, K. M.; Monard, G.; Needham, P.; Nguyen, H.; Nguyen, H. T.; Omelyan, I.; Onufriev, A.; Roe, D. R.; Roitberg, A.; Salomon-Ferrer, R.; Simmerling, C. L.; Smith, W.; Swails, J.; Walker, R. C.; Wang, J., Wolf, R. M.; Wu, X.; York, D. M.; Kollman, P. A. AMBER 2015; University of California: San Francisco, USA, 2015.

(8) Maier, J. A.; Martinez, C.; Kasavajhala, K., Wickstrom, L.; Hauser, K. E.; Simmerling, C. ff14SB: Improving the Accuracy of Protein Side Chain and Backbone Parameters from ff99SB. $J$. Chem. Theory Comput. 2015, 11, 3696-3713. 